\title{
Comparative Analysis of Repeat Biopsies in Patients with Isolated High-grade Prostatic Intraepithelial Neoplasia in Their First Prostate Needle Biopsies of 6 Versus 12 Cores and Systematic Literature Review
}

\author{
(1) Gupse TURAN, (1) Sevgiye KAÇAR ÖZKARA \\ Department of Pathology, Kocaeli University Faculty of Medicine, Kocaeli-Turkey
}

\begin{abstract}
OBJECTIVE
The objective of the study was to investigate the relationship between histopathological features of isolated high-grade prostatic intraepithelial neoplasia (HGPIN) and carcinoma risk in repeat prostate needle biopsies of 6 versus 12 cores.
\end{abstract}

\section{METHODS}

Of 114 isolated HGPIN cases were included; 64 with 6 ( $1^{\text {st }}$ group) and 50 with 12 cores ( $2^{\text {nd }}$ group). The number of cores with HGPIN, laterality, structural PIN subtypes, prominent nucleolus, and nuclear pleomorphism was examined in the first and follow-up biopsies. The relationship between the results of follow-up biopsies and clinicopathological findings was evaluated statistically. Furthermore, a systematic literature review was carried out.

\section{RESULTS}

Carcinoma was found in repeat biopsies in $15.6 \%$ of the cases in 6 -core and $24 \%$ of cases with 12 -core biopsies $(\mathrm{p}=0.006)$. Carcinoma detection rate was significantly higher in cases with $\geq 2$ cores with HGPIN than in cases with a single core of HGPIN ( $\mathrm{p}=0.007)$. The rates of carcinoma and persistent HGPIN were higher in multifocal and bilateral HGPIN cases, compared to unifocal, or multifocal but unilateral cases $(\mathrm{p}=0.018)$. In both groups, prominent nuclear pleomorphism was significantly more common in cases with carcinoma $(\mathrm{p}=0.023)$. The systematic literature review revealed $25.6 \%$ of carcinoma risk out of 5580 isolated HGPIN patients.

\section{CONCLUSION}

Carcinoma detection rate in repeat biopsies of 12-cores was significantly higher than cases with 6-core biopsies. The HGPIN cases with $\geq 2$ cores, bilaterality, and presence of prominent nuclear pleomorphism were found to be significant histopathological markers in predicting patients with carcinoma. Close follow-up of high risk patients with repeat biopsies in addition to clinical parameters was recommended.

Keywords: Bilateral; carcinoma; high grade prostatic intraepithelial neoplasia; multifocal; prostate; prostatic intraepithelial neoplasia.

Copyright $\odot$ 2021, Turkish Society for Radiation Oncology

Dr. Sevgiye KAÇAR ÖZKARA

Kocaeli Üniversitesi Tıp Fakültesi,

Patoloji Anabilim Dalı,

Kocaeli-Turkey

E-mail:emrecan1999@yahoo.com 


\section{Introduction}

Prostate cancer is the second most common cancer in men; it ranks fifth among cancer deaths in men.[1] Its incidence increases with age. Digital rectal examination (DRE) is a frequently recommended method in prostate cancer screening; however, it is not sensitive in detecting early disease. Although serum prostate specific antigen (sPSA) screening is widely accepted; it does not have a high sensitivity and specificity for prostate cancer due to reasons such as the increase in sPSA levels with age and prostate cancer can also be seen in men with low sPSA levels.[2] The standard method for detecting prostate cancer is prostate needle biopsy with transrectal ultrasonography.

Prostatic intraepithelial neoplasia (PIN) is a histopathological lesion that accompanies prostate cancer and is considered a cancer precursor. It is defined as the limited proliferation of the secretory cells of the ductal-acinar system of the prostate within the gland and is characterized by the presence of atypical cytological features. McNeal and Bostwick first emphasized the potential malignant nature of this proliferative change in 1965, and in 1986 they defined the diagnostic criteria with Bostwick. [3,4] PIN is graded in two groups, high grade and low grade, and only high grade PIN (HGPIN) is associated with prostatic adenocarcinoma.[5] Its presence in the biopsy specimens is considered to be a warning sign for the possibility of carcinoma that may be detected simultaneously or later. It does not make a palpable mass and cannot be detected by ultrasonography; the only diagnostic method of HGPIN is histopathological examination. [6]

The incidence of isolated HGPIN in initial biopsies ranges from $0.6 \%$ to $24.6 \%$.[7-11] Most of the studies on the effectiveness of isolated HGPIN diagnosis in predicting prostate cancer include 6-core biopsy protocols. The probability of detecting prostate cancer in repeat biopsies performed after HGPIN varies between $25 \%$ and $79 \%$ in cases where the 6-core biopsy technique is applied.[7,8,11-17] As more sampling-based biopsy protocols began to replace classical six quadrant biopsies, the rates of cancer detection in repeat biopsies after HGPIN generally decreased. In the recent series, the probability of detecting prostate cancer as a result of repeat biopsies has been found to be around $20 \%$ in patients who underwent prostate biopsy with samples of 10 cores or more and were diagnosed with HGPIN.[7,11]

This study including cases with isolated HGPIN detected in prostate needle biopsies evaluated in two periods between 1996 and 2005 when 6-core proto- col was applied and 2006-2018 when 12-core protocol was applied in our institution. In patients diagnosed with isolated HGPIN with one or more follow-up biopsies in prostate needle biopsies in both periods, the relationship between the foci of HGPIN and their histopathological features with the development of carcinoma was investigated. It was aimed to compare the clinicopathological findings of the cases of these two periods in which 6-core and 12-core needle biopsy protocols were applied. At the same time, a systematic literature review was conducted to compile the results of the largest published case series on this subject.

\section{Materials and Methods}

The approval of Institutional Non-Invasive Research Ethics Committee was obtained (GOAEK 2020/15.09; Project number: 2020/260) for this study.

At the first step, 6-core prostate needle biopsies evaluated between January 1, 1996, and December 31, 2005 , were reviewed in the report archive of our department. At the second step, 12-core prostate needle biopsies evaluated and reported between January 1, 2006, and December 31, 2018, were reviewed. Cases with adenocarcinoma, isolated HGPIN without repeat biopsy, atypical small acinar proliferation (ASAP), and benign prostate tissue (BPT) were excluded from the study; and only isolated HGPIN cases with at least one repeat biopsy were included into the study (Fig. 1). Follow-up periods between the first and last biopsies were recorded. The cases belonging to the first period were coded as Group I, and the cases belonging to the second period as Group II.

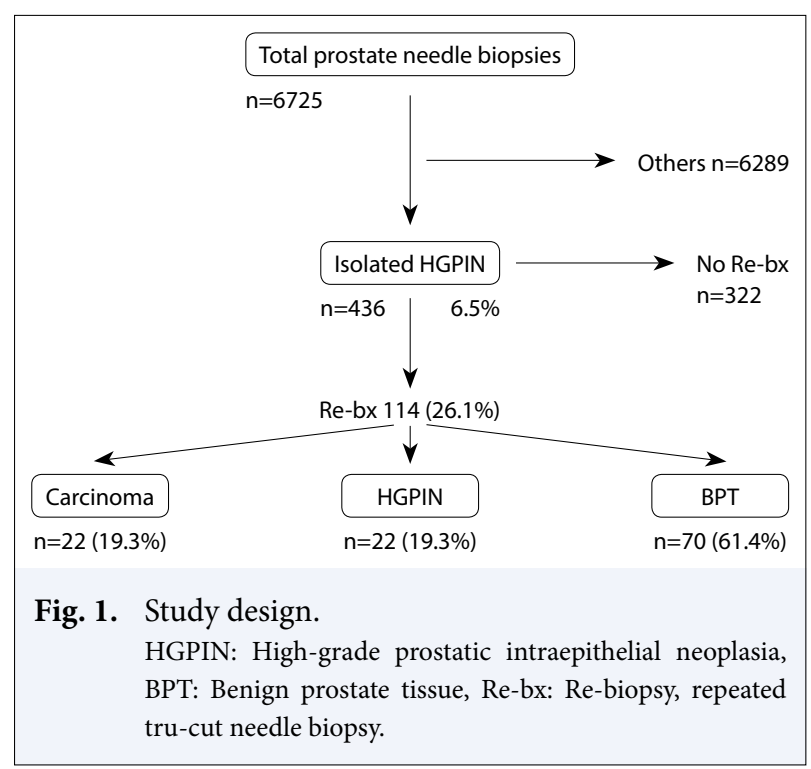


Hematoxylin-Eosin stained slides of both initial and follow-up biopsies of each case were re-evaluated according to the standard histopathological criteria accepted for the diagnosis of HGPIN under light microscopy (Olympus BX50).[8,12] For each patient, the number of cores showing HGPIN was recorded in baseline and follow-up biopsies. Since the number of cores may be variable in biopsies, the positive core ratio obtained by dividing the number of positive cores by the total number of cores was also calculated for each biopsy. Linear lengths of all cores and foci with HGPIN in the cases diagnosed with HGPIN in both initial and follow-up biopsies were measured with an ocular micrometer, and the ratio of HGPIN foci in the whole biopsy sample was calculated. The total number of glands with HGPIN and the number of glands with HGPIN for each biopsy was counted, and the ratio of the number of glands with HGPIN to the number of glands in the whole biopsy sample was calculated. The ratio of involvement in glands retained with HGPIN was evaluated according to the extent of intra-glandular PIN for each biopsy and whether it involves the entire gland. The structural PIN subtype(s) seen in each biopsy were recorded. The degree of nucleolus prominence in glands with PIN was examined in two groups according to the presence of glands containing prominent nucleoli at $\times 200$ and $\times 400$ magnification. Histopathological findings such as the presence of prominent nuclear pleomorphism in the foci of HGPIN, the presence of inflammation and atrophic glands, mitotic activity, mucin, and crystalloid presence were also recorded. The results of the follow-up biopsies of the cases were categorized into three groups as BPT, HGPIN, and carcinoma.

The relationship of histopathological findings within the groups was evaluated using the Statistical Package for the Social Sciences 25.0 package program statistically using Monte Carlo Chi-square and independent $\mathrm{t}$-tests. If $\mathrm{p}<0.05$, the difference between the groups was considered significant. Continuous and categorical variables were defined as mean \pm standard deviation and percentage, respectively.

In addition, a systematic literature review was conducted, including reports of the largest case series (consisting of at least 100 cases of HGPIN having correlation results with repeated biopsies). The PubMed database was searched for the relevant literature between 1990 and 2020. Search terms were "prostate" and "needle biopsy" and "HGPIN." In addition, a manual search was performed by reviewing the relevant publications presented in PubMed and the citations in the related references. Additional articles were found or removed by manually reviewing the full text and references of the matching articles. To identify relevant observational studies, inclusion criteria were as follows: (1) An observational study design; (2) the definition of the HGPIN group and the number of cases clearly stated; (3) the final histopathological malignancy rate in the HGPIN group was reported; and (4) studies written in English as a full text publication. Relevant results, including the total number of prostate needle biopsy, total number of HGPIN cases, the rate of diagnosis of HGPIN from prostate needle biopsy, and the rate of final histopathological malignancy diagnosis in HGPIN, were reviewed and analyzed in the articles included into the study.

\section{Results}

Out of 6725 prostate needle biopsy cases evaluated in our department between 1996 and 2018, a total of 436 (6.5\%) were diagnosed with isolated HGPIN. Out of these isolated HGPIN cases, 114 (26.1\%) patients had at least one repeat biopsy and constituted the target study group (Fig. 1). Carcinoma was detected in 19.3\% of these cases in repeat biopsies, while the diagnosis of HGPIN persisted in $19.3 \%$ and $61.4 \%$ was diagnosed with BPT (Table 1).

\section{Group I Cases (With 6-Core Biopsy Protocol)}

When the 6-core biopsy protocol was applied between January 1, 1996, and December 31, 2005, a total of 1402 prostate needle biopsies were evaluated. Adenocarcinoma was diagnosed in 399 cases (28.4\%), and isolated HGPIN was diagnosed in 211 (15\%); 17 were diagnosed with isolated ASAP (1.2\%), and 775 (55.2\%) were diagnosed with BPT (Table 1). It was found that

\begin{tabular}{lccc} 
Table $\mathbf{1}$ & \multicolumn{3}{l}{$\begin{array}{l}\text { The distribution of histopathological diagnoses } \\
\text { in prostate needle biopsies with 6-core (1996- } \\
\text { 2005) and 12-core (2006-2018) protocols }\end{array}$} \\
$\begin{array}{lccc}\text { Histopathological } \\
\text { diagnoses }\end{array}$ & $\begin{array}{c}\text { 6-core } \\
\text { Group I } \\
\text { n (\%) }\end{array}$ & $\begin{array}{c}\text { 12-core } \\
\text { Group II } \\
\mathbf{n}(\%)\end{array}$ & $\begin{array}{c}\text { Total } \\
\mathbf{n}(\%)\end{array}$ \\
\hline $\begin{array}{l}\text { Adenocarcinoma } \\
\text { HGPIN }\end{array}$ & $399(28.4)$ & $2038(38.3)$ & $2437(36.2)$ \\
BPT & $\mathbf{2 1 1 ( 1 5 . 0 )}$ & $\mathbf{2 2 5 ( 4 . 2 )}$ & $\mathbf{4 3 6 ( 6 . 5 )}$ \\
ASAP & $775(55.2)$ & $2987(56.1)$ & $3762(55.9)$ \\
Total & $17(1.2)$ & $73(1.4)$ & $90(1.3)$ \\
\hline
\end{tabular}

Bold text indicates statistical significance. HGPIN: High-grade prostatic intraepithelial neoplasia; BPT: Benign prostate tissue; ASAP: Atypical small acinar proliferation 
64 of the cases diagnosed with isolated HGPIN had repeat biopsies during their follow-up (Table 2).

Patient age ranged from 48 to 86 years in 64 isolated HGPIN cases in the first group; the mean patient age was $64.4(\mathrm{SD}=8.743)$. sPSA levels before the first biopsies ranged from $1.1 \mathrm{ng} / \mathrm{ml}$ to $20 \mathrm{ng} / \mathrm{ml}$; averaging $7.1 \mathrm{ng} / \mathrm{ml}$. The follow-up period of the patients varied between 1 month and 54 months, and the average follow-up period was 12.4 months.

Carcinoma was detected in ten cases (15.6\%) at repeat biopsies; the presence of isolated HGPIN continued in 19 cases (29.7\%) and 35 cases (54.7\%) was diagnosed with BPT (Table 2). Gleason pattern score distribution in cases with carcinoma in repeat biopsies was: Gleason pattern score 6 (Gleason grade Group 1) in 8 patients ( $80 \%)$; the Gleason pattern score was 7 (Gleason grade Group 2) in 1 patient (10\%); and Gleason pattern score 8 (Gleason grade Group 4) in another (10\%) patient (Table 2 and Fig. 2a).

Multifocal HGPIN was present in $50 \%$ of the cases in the first group. The ratio of cases with bilateral HGPIN was also higher (37.5\%) in the first group. On the other hand, the ratio of cases with carcinoma detected at repeat biopsies was lower (15.6\%) than the expected range in the first group.

The rates of carcinoma and persistent HGPIN were higher in multifocal and bilateral HGPIN cases compared to unifocal or multifocal but unilateral cases $(\mathrm{p}=0.018)$ (Table 3).

The difference between the diagnostic groups was statistically significant $(\mathrm{p}=0.023)$ in terms of the presence of significant nuclear pleomorphism in the glands in which HGPIN was detected in the first biopsies.
The frequency of marked pleomorphism observed in the group diagnosed with BPT on repeat biopsies was much lower than the other groups. The distribution of the presence of prominent nuclear pleomorphism according to the diagnostic groups is shown in Table 3.

No statistically significant difference was found between the groups with and without carcinoma development in repeat biopsies in terms of the ratio of cores showing involvement with HGPIN in both the first and repeat biopsies, the extent and number of glands containing HGPIN in each core and the ratio of involvement in the gland $(\mathrm{p}>0.05)$.

There was no statistically significant difference between the groups with and without carcinoma in terms of HGPIN pattern type either ( $p>0.05)$. Considering the types of PIN patterns detected in both the first and second biopsies, a single type (pure) pattern was observed in $65.3 \%$ of the cases, and multiple (composite) HGPIN patterns were observed in $34.7 \%$ of the cases. One type of PIN pattern was observed in all cases with carcinoma on repeat biopsy. $(\mathrm{p}=0.013)$.

\section{Group II Cases (With 12-Core Biopsy Protocol)}

During the 13-year period when 12-core biopsy protocol was applied between 2006 and 2018, 2038 out of 5323 prostate needle biopsies evaluated in our department were diagnosed as adenocarcinoma (38.3\%), 225 as isolated HGPIN (4.2\%), 73 as ASAP (1.4\%), and 2987 (56.1\%) was diagnosed with BPT (Table 1). Fifty of the cases diagnosed with isolated HGPIN had at least one repeat biopsy (Table 2).

In the cases of the second group, the patient's age ranged from 55 to 94 ; mean patient age was 73.9

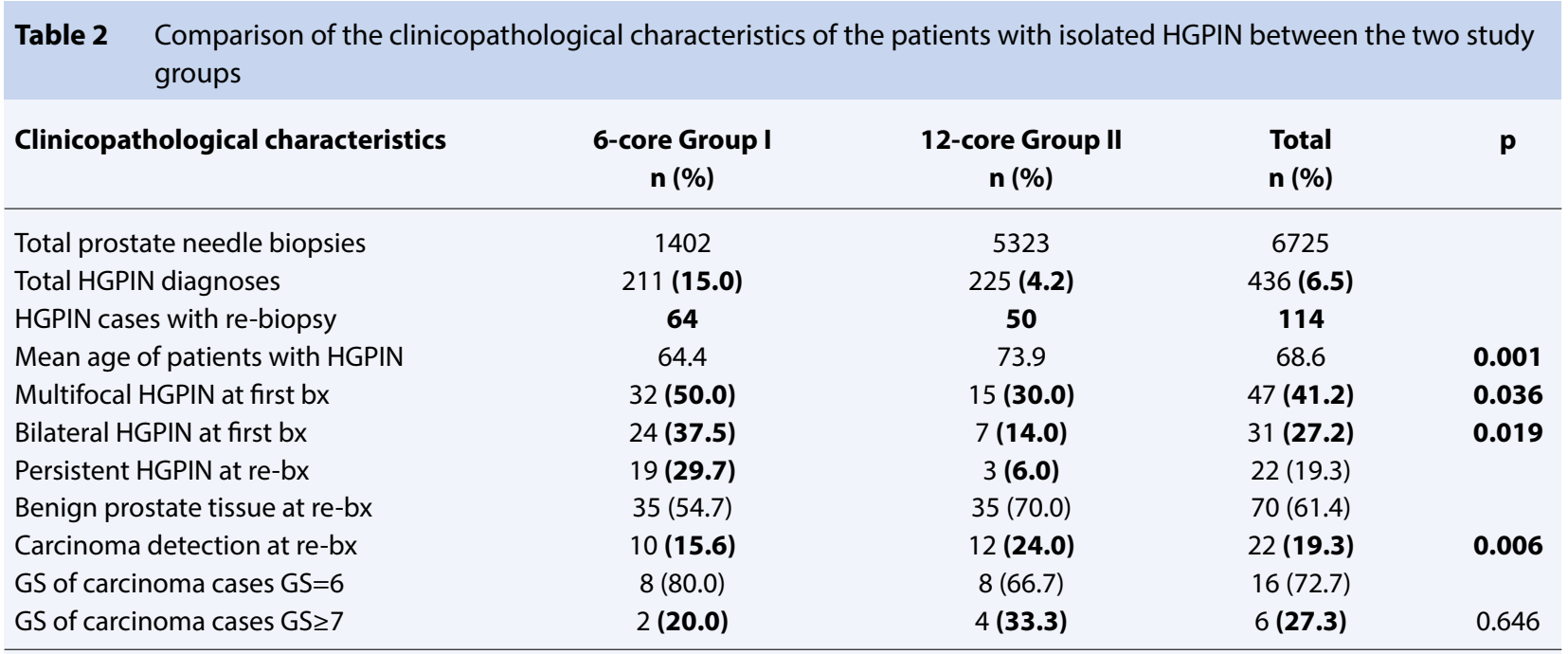

Bold text indicates statistical significance. HGPIN: High-grade prostatic intraepithelial neoplasia; GS: Gleason score; Re-bx: Re-biopsy, repeated tru-cut needle biopsy 
$(\mathrm{SD}=7.785)$. The age distribution of patients with carcinoma on repeat biopsy varied between 50 and 90 (mean 70.9; SD: 10.389). No statistically significant difference was found between the patient groups with and without carcinoma on repeat biopsies in terms of mean patient age ( $\mathrm{p}>0.05)$.

sPSA levels before the first biopsies ranged from 0.3 $\mathrm{ng} / \mathrm{mL}$ to $44.2 \mathrm{ng} / \mathrm{mL}$; it averaged $9.6 \mathrm{ng} / \mathrm{mL}$. The follow-up period of the patients varied between 1 month and 107 months, and the average follow-up period was 28.7 months.

Carcinoma was detected in 12 cases $(24 \%)$ at repeat biopsies. Isolated HGPIN presence continued in three cases (6\%), and 35 cases $(70 \%)$ were diagnosed with BPT (Table 2). Gleason pattern score distribution in cases with carcinoma at repeat biopsies was: Gleason pattern score 6, (Gleason grade Group 1) in 8 patients (66.7\%); Gleason pattern score 7 (Gleason grade Group 2) in two patients (16.7\%); Gleason pattern score 8, (Gleason grade Group 4) in one patient (8.3\%); and the Gleason pattern score was 9, (Gleason grade Group 5) in one patient (8.3\%) (Table 2 and Fig. 2b).

Multifocal HGPIN was present in $30 \%$ of the cases and the ratio of cases with bilateral HGPIN was also lower (14\%) in the second group. On the other hand, the ratio of cases with carcinoma detected in repeat biopsies was higher (24\%) in the second group. In multifocal and bilateral HGPIN cases, the rates of carcinoma and persistent HGPIN were higher than multifocal but unilateral or unifocal cases $(\mathrm{p}=0.018)$ (Table 3).

The difference between the diagnostic groups was statistically significant $(\mathrm{p}=0.023)$ in terms of the presence of significant nuclear pleomorphism in the glands with HGPIN detected in their first biopsies. The frequency of marked pleomorphism observed in the group diagnosed with BPT in the final biopsy was much lower than the other groups. The distribution of the presence of prominent pleomorphism by diagnostic groups is shown in Table 3.

There was no statistically significant difference between the groups with and without carcinoma development at repeat biopsies in terms of the ratio of cores that showed involvement with HGPIN in both the first and repeat biopsies, the extent and number of glands containing HGPIN in each core, and the ratio of involvement in the gland. There was no statistically significant difference between the groups with and without carcinoma in terms of HGPIN pattern type either $(\mathrm{p}>0.05)$.

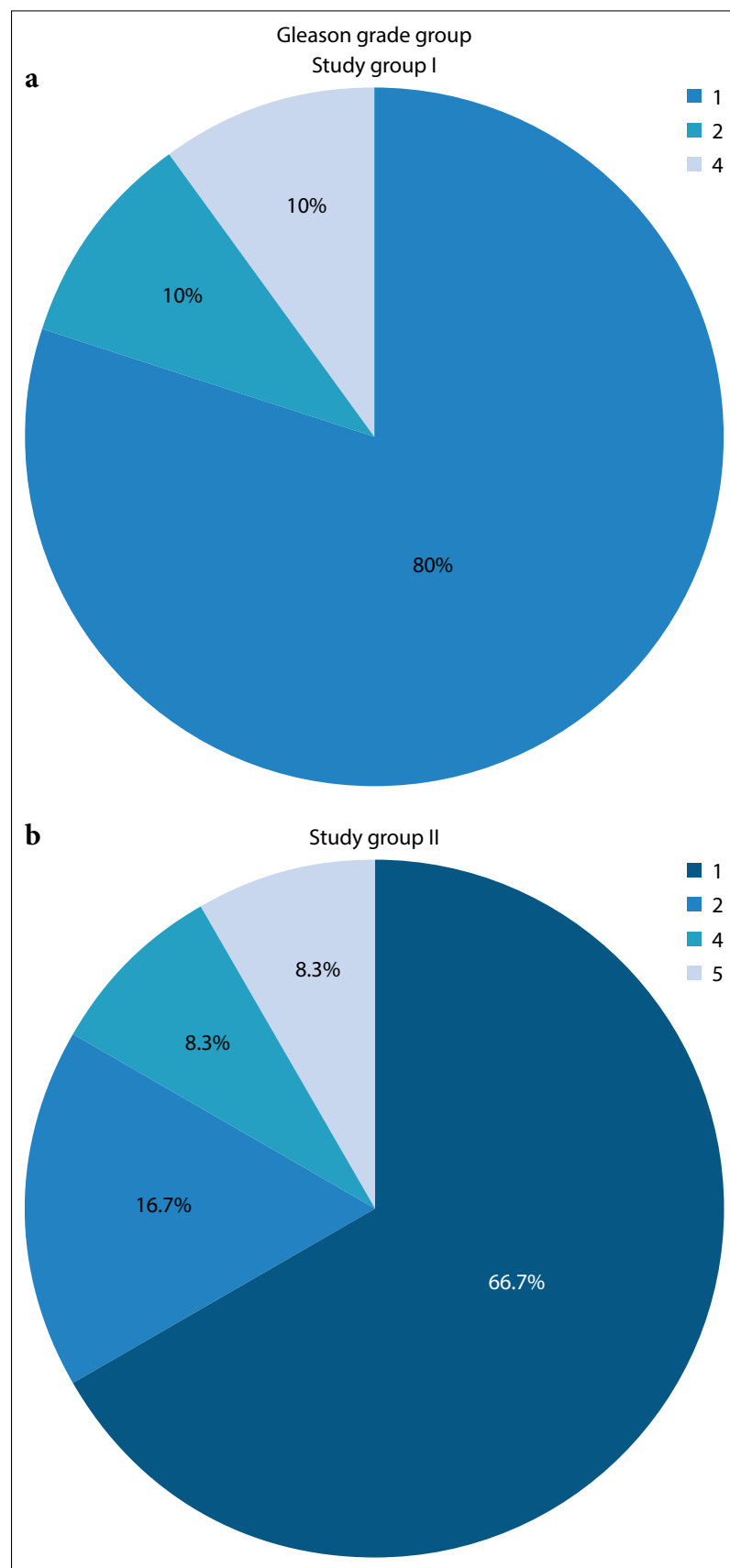

Fig. 2. (a, b) Gleason grade group distribution of tumors in carcinoma cases at repeat biopsies in the study groups I (a: 6-cores; $\mathrm{n}_{1}=10$ ) and II (b: 12-cores; $\left.\mathrm{n}_{2}=12\right)$.

\section{Comparison of the Findings of the Cases between the Study Groups I and II}

While $28.4 \%$ of the cases in the first period in which the 6-core biopsy protocol was applied were diagnosed with adenocarcinoma, it was observed that the rate of adenocarcinoma diagnosis increased to $38.3 \%$ in the 
Table 3 Distribution of repeat biopsy diagnoses according to the number of cores with HGPIN, core foci with HGPIN and laterality status and the presence of prominent nuclear pleomorphisma in the first biopsy samples with isolated HGPIN

\begin{tabular}{|c|c|c|c|c|c|}
\hline Cores with HGPIN in the first biopsy & $\begin{array}{c}\text { Carcinoma } \\
n(\%)\end{array}$ & $\begin{array}{l}\text { HGPIN } \\
\text { n (\%) }\end{array}$ & $\begin{array}{c}\text { BPT } \\
\text { n (\%) }\end{array}$ & $\begin{array}{l}\text { Total } \\
\text { n (\%) }\end{array}$ & $\mathbf{p}$ \\
\hline 1 core with HGPIN & $10(14.9)$ & $8(11.9)$ & 49 (73.1) & $67(100)$ & \\
\hline$\geq 2$ cores with HGPIN & $12(\mathbf{2 5 . 5})$ & $14(29.8)$ & $21(44.7)$ & $47(100)$ & 0.007 \\
\hline Unifocal/Unilateral HGPIN & $10(14.9)$ & $8(11.9)$ & 49 (73.1) & $67(100)$ & \\
\hline Multifocal/Unilateral HGPIN & $4(25.0)$ & $3(18.8)$ & $9(56.3)$ & $16(100)$ & \\
\hline Multifocal/Bilateral HGPIN & $8(25.8)$ & 11 (35.5) & $12(38.7)$ & $31(100)$ & 0.018 \\
\hline Prominent nuclear pleomorphism present ${ }^{\mathrm{a}}$ & $15(\mathbf{2 8 . 9 )}$ & $13(25.9)$ & $24(46.2)$ & $52(100)$ & \\
\hline Prominent nuclear pleomorphism absent ${ }^{\mathrm{a}}$ & $6(13.3)$ & $3(6.7)$ & $36(80.0)$ & $45(100)$ & 0.023 \\
\hline Total & $22(19.3)$ & $22(19.3)$ & $70(61.4)$ & $114(100)$ & \\
\hline
\end{tabular}

Bold text indicates statistical significance. ${ }^{\text {a: }}$ Slides of 97 cases were re-evaluated. HGPIN: High-grade prostatic intraepithelial neoplasia; BPT: Benign prostate tissue

second period when the 12-core protocol was applied. While the rate of HGPIN diagnosis in 6-core biopsies was $15.0 \%$, we observed that the rate of HGPIN in 12 core biopsies decreased to $4.2 \%$. We did not see any significant changes in other diagnostic groups (i.e., BPT and ASAP).

Multifocal HGPIN was present in 50\% of the cases in the first group and in $30 \%$ of the cases in the second group, the ratio of cases with bilateral HGPIN was also higher in the first group. On the other hand, the ratio of cases with carcinoma detected at repeat biopsies was higher $(24 \%)$ in the second group than that (15.6\%) in the first group.

Carcinoma was detected in repeat biopsies in 10 (15.6\%) of the cases in the first group (6 cores) and in 12 cases $(24 \%)$ with 12 -core biopsies $(\mathrm{p}=0.006)$ (Table 2 ). The rate of carcinoma detection in repeat biopsies was found to be significantly higher in patients with $\geq 2$ cores with HGPIN than the patients with single-core HGPIN ( $\mathrm{p}=0.007$ ) (Table 3). The rates of carcinoma and persistent HGPIN were higher in multifocal and bilateral HGPIN cases compared to unifocal or multifocal but unilateral cases $(\mathrm{p}=0.018)$. In both 6 and 12 -core biopsy groups, the presence of prominent nuclear pleomorphism in the glands with HGPIN was significantly more common in cases with carcinoma at repeat biopsy $(\mathrm{p}=0.023)$ (Table 3$)$. No significant correlation was found with other histopathological findings ( $>0.05)$. Examples of our cases showing different PIN patterns are presented in Figure 3a-d.

\section{Systematic Literature Review}

Our systematic review of the literature revealed an overall malignancy rate of $25.6 \%$ (ranging from $13 \%$ to $38 \%$ ) among the largest studies of at least 100 cases with a diagnosis of isolated HGPIN and with re-biopsy correlation results. In addition, significant variations were observed in the use of diagnosis of HGPIN in different centers with reporting rates varying between $1 \%$ and $10 \%$. The studies included (a total of 5580 cases with repeat biopsy correlation in the systematic analysis of the largest case series) are by Herawi et al.,[18] El-Fakharany et al.,[19] Merrimen et al.,[6] Sakr et al.,[20] Girasole et al.,[9] Lee et al.,[21] Roscigno et al.,[22] Pierson et al.,[23] Kronz et al.,[12] Eskicorapci et al.,[24] Schlesinger et al.,[25] Gokden et al.,[8] Kim et al.,[10] Siever et al.,[26] Bishara et al.,[7] O'dowd et al.,[27] Sakr et al.,[20] Postma et al.,[28] Davidson et al.,[29] and Ongun et al.[11] They are listed in Table 4, in the same order as descending case numbers, with the data reported by them. Six of these series (by El-Fakharany, Sakr, Pierson, Siever, and Sakr et al.) were presented only as abstracts and the full text of these studies could not be reached. Because they were referred in the previous publications, [25] they are included into the systematic analysis.

\section{Discussion}

The role of HGPIN as a risk factor for prostate carcinoma is not sufficiently elucidated from the pathologist's perspective. Although HGPIN is genetically related to prostate carcinoma, a wide range from $2 \%$ to $100 \%$ has been reported in the literature, which cannot be thoroughly interpreted in terms of prostate carcinoma incidence after the diagnosis of isolated HGPIN. The problem is further complicated by the transi- 


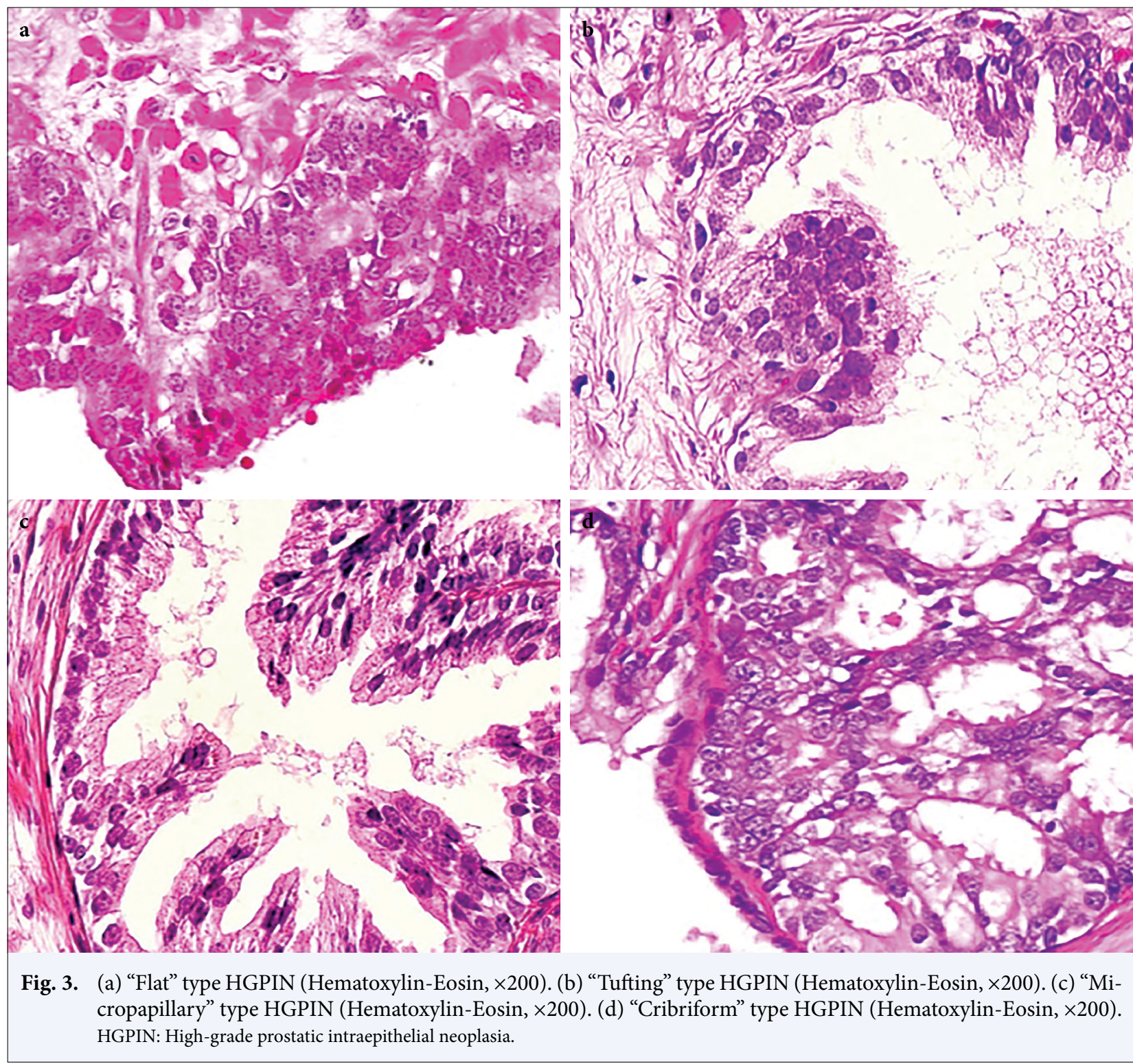

tion from sextant biopsies (with 6-cores) to extended biopsy (with $\geq 12$-cores) protocols $[6,17,21,25]$ and has not been re-evaluated recently.

The clinical significance of HGPIN is related to its predictive value as a marker for prostate carcinoma. In different studies conducted in large case series from 1990 to 2020, the incidence of isolated HGPIN detected in prostate needle biopsies varies in a wide range between $0.6 \%$ and $24.6 \%$. The average frequency of HGPIN is $7.7 \%$, with a median of $5.2 \%$.[4,30-32] The $6.5 \%$ isolated HGPIN rate we found in our study is also around the general average.

After the initial diagnosis of HGPIN, the rate of prostate carcinoma detection has dropped from $50 \%$ to
$40 \%$ in the early 1990 s to $35-20 \%$ in later studies. The change in prostate sampling from the sextant to the extended or dual sextant protocol has been thought to be largely responsible for this reduction. $[6,22]$ On the other hand, the probability of detecting prostate cancer in repeat biopsies of patients whose first prostate biopsy is evaluated as benign is around $10-20 \%$.[6,11,33] It has been discussed by Eskicorapci et al. and Gokden et al. $[8,24]$ that HGPIN is a risk factor for carcinoma detection only when found in sequential sextant biopsies. Their presented data raise the re-evaluation of biopsy strategies after the diagnosis of isolated HGPIN and requestioning the absolute necessity of repeat biopsy for all patients with HGPIN. 
Table 4 Carcinoma detection rates at repeat biopsies of cases in studies consisting of large case series $\geq 100$ patients in which the first prostate needle biopsy was diagnosed as isolated HGPIN

\begin{tabular}{|c|c|c|c|}
\hline Study/First author, year & $\begin{array}{c}\text { Total } \\
\text { HGPIN } \\
\text { with } \\
\text { re-Bx }\end{array}$ & $\begin{array}{c}\text { Carcinoma } \\
\text { at re-Bx }\end{array}$ & $\begin{array}{c}\text { Malignancy } \\
\text { risk (\%) }\end{array}$ \\
\hline Herawi et al., 2006[18] & 791 & 139 & 17.6 \\
\hline El-Fakharany et al., 2005[19] & 585 & 196 & 33.5 \\
\hline Merrimen et al., 2009[6] & 564 & 155 & 27.5 \\
\hline Sakr et al., 2003[20] & 540 & 146 & 27.0 \\
\hline Girasole et al., 2006[9] & 358 & 79 & 22.1 \\
\hline Lee et al., 2011[21] & 314 & 77 & 24.5 \\
\hline Roscigno et al., 2012[22] & 262 & 83 & 31.7 \\
\hline Pierson et al., 2004[23] & 249 & 52 & 21.0 \\
\hline Kronz et al., 2001[12] & 245 & 79 & 32.2 \\
\hline Eskicorapci et al., 2007[24] & 211 & 54 & 25.6 \\
\hline Schlesinger et al., 2005[25] & 204 & 47 & 23.0 \\
\hline Gokden et al., 2005[8] & 190 & 58 & 30.5 \\
\hline Kim et al., 2015[10] & 149 & 33 & 22.1 \\
\hline Siever et al., 2003[26] & 145 & 36 & 25.0 \\
\hline Bishara et al., 2001[7] & 132 & 38 & 28.8 \\
\hline O'dowd et al., 2000[27] & 125 & 28 & 22.6 \\
\hline Turan\&Özkara 2021 & 114 & 22 & 19.3 \\
\hline Sakr et al., 2003[20] & 101 & 38 & 38.0 \\
\hline Postma et al., 2004[28] & 101 & 13 & 13.0 \\
\hline Davidson et al., 1996[29] & 100 & 35 & 35.0 \\
\hline Ongun et al., 2016[11] & 100 & 20 & 20.0 \\
\hline Total & 5580 & 1428 & 25.6 \\
\hline
\end{tabular}

HGPIN: High-grade prostatic intraepithelial neoplasia; Re-bx: Re-biopsy, repeated tru-cut needle biopsy

The lower rates of carcinoma detection in repeat biopsies performed following the diagnosis of isolated HGPIN have been thought to be the result of the biopsy approach aimed at more expanded sampling (mean 12 cores).[18,34] According to this, in the first biopsies taken with the 6-core biopsy technique, small and low-stage cancers accompanying HGPIN are probably missed and the cases are diagnosed with isolated HGPIN. The possibility of detecting cancer accompanying HGPIN increases when the $\geq 12$ core biopsy approach is applied, which aims sampling more widely in repeat biopsies after the first biopsy. In cases where $\geq 12$ cores are sampled in the first biopsy, existing cancer foci can be detected with a higher probability. Therefore, the diagnosis of isolated HGPIN given in the first biopsies with 12 cores is more reliable than 6-core biopsies. In this case, it is expected that the probability of detecting carcinoma is low in repeat biopsies in cases where $\geq 12$ cores are sampled. In the first biopsies in which 12 or more cores are sampled, it becomes easier to detect cancer associated with HGPIN and the possibility of misleading isolated HGPIN diagnosis is reduced. Thus, cancers detected in follow-up biopsies represent cancers that develop after isolated diagnosis of HGPIN. Today, this is accepted as the main reason why cancer rates detected in repeat biopsies after diagnosis of HGPIN is lower than in the previous years.[18]

The probable reason for the higher incidence rates of isolated HGPIN detected in the first group of our study is that the biopsies we evaluated were sampled with the 6-core method. In 2006, the standard sampling method with $\geq 12$ cores started to be used in prostate needle biopsies in our hospital. In the first group, the first biopsies of all ten patients with carcinoma on repeat biopsy were performed before 2006, and the number of cores sampled in the first and repeat biopsies of the patients was 6. However, in our study, while the frequency of isolated HGPIN cases diagnosed after the standard 12-core sampling method in 2006 and later decreased as expected; the rate of carcinoma detected on repeat biopsies has increased and our results partially support the accuracy of the approach described above. We think that the significant difference between the mean ages of the two groups might also be important in this result.

Most of the studies did not report the total number of patients diagnosed with isolated HGPIN and the Gleason score of carcinomas detected on followup biopsies during the study period. Girasole et al.[9] reviewed 42.667 biopsies from a urological pathology reference laboratory, and identified isolated HGPIN in the first biopsies taken from 1293 patients (3\%). Many articles have been written about the optimum timing and technique for re-biopsy after the initial diagnosis of HGPIN. However, there is no contemporary consensus on the management of patients with HGPIN. [10] The current study sought to determine the risk of cancer after an initial diagnosis of HGPIN and ascertain whether histopathologic findings can help predict which patients are at increased risk of having cancer on repeat biopsy. Because, additional factors are needed to determine if there are subgroups of patients with HGPIN who have a greater risk of being subsequently diagnosed with cancer.

Merrimen et al.[6] reported in 2009, a higher likelihood of prostate carcinoma when multiple prostatic sites ( $\geq 2$ cores) were involved. A year later, Lee et al.[35] published that both multifocal and bilateral HGPIN confer an increased risk for the future detection of prostate carcinoma with estimated 3-year cancer rates 
of $29 \%$ and $37 \%$, respectively. By contrast, isolated unifocal HGPIN on initial biopsy conferred no additional risk to the detection of prostate carcinoma compared with benign disease. In their later study, both multifocal HGPIN on initial biopsy as well as number of cores on second biopsy were independently associated for the detection of prostate carcinoma on repeat biopsy after adjusting for age.[21]

Afterward, Roscigno et al.[22] developed for the $1^{\text {st }}$ time a predictive nomogram that could be helpful for patient counseling and to guide the clinician to perform re-biopsy after an initial diagnosis of isolated HGPIN. In their study, prostate carcinoma detection was significantly higher in patients with a $\leq 12$-core initial prostate biopsy than in those with a $>12$-core initial prostate biopsy, as well as in patients with multifocal HGPIN than in those with unifocal HGPIN. At multivariable analysis, PSA level, age, multifocal HGPIN, and $\leq 12$-core initial prostate biopsy were independent predictors of prostate carcinoma detection. We also found that the presence of multifocal and bilateral HGPIN and prominent nuclear pleomorphism in the initial prostate needle biopsy was significantly associated with higher likelyhood of carcinoma detection at the repeat biopsy and carcinoma detection rate did not decrease after the routine usage of 12-core protocol in our institution.

All the above data including ours, demonstrates the potentially dangerous dilutional effect of labeling HGPIN as a single entity, and might explain why HGPIN without stratifying it into unifocal or multifocal disease was not a predictor of prostate carcinoma in many studies.

From the histomorphological point of view, the only detailed histopathological study in the literature that questions the relationship between the presence of prominent nuclear pleomorphism and carcinoma is made by Kronz et al. In their series of 245 cases, cancer was detected in $33 \%$ of the follow-up biopsies of HGPIN cases with distinctly pleomorphic nuclei. However, in their study, a statistically significant correlation could not be demonstrated between the presence of prominent nuclear pleomorphism and detection of carcinoma at repeat biopsies in patients with HGPIN. The detailed histopathologic examination by Kronz et al.[12] also showed that the following features did not predict cancer on the first repeat biopsy: Number of HGPIN., glands, maximum percentage of gland involved by HGPIN., nucleolar prominence, percentage of PIN cells with prominent nucleoli, pattern of HGPIN (flat, tuft, micropapillary, and cribriform), and marked pleomorphism. The only histopathologic finding that predicted cancer was the number of cores involved by HGPIN.

In our study, the frequency of significant nuclear pleomorphism observed in the glands with HGPIN in their first biopsies was significantly lower in the group diagnosed with BPT in follow-up biopsies compared to other diagnostic groups. The frequency of prominent nuclear pleomorphism in the first biopsies of cases with persistent HGPIN and carcinoma in repeat biopsies was significantly higher than in cases with BPT in repeat biopsies.

In the publications examining the relationship between the structural pattern in glands with HGPIN and the risk of carcinoma in follow-up biopsy, the structural type of the observed patterns was taken as a basis, and the relationship between the pure or compound pattern and the risk of carcinoma was not evaluated separately. In our study, all of the pure and compound patterns observed in the first and second biopsies were evaluated in detail of pattern types and frequency; it was coded according to both the type of structural pattern and whether the pattern observed was pure or compound. When the type of structural PIN pattern was examined, no significant difference was found between the groups with and without cancer at followup. In the first group, it was noteworthy that pure PIN pattern was observed in all cases with tumors found in follow-up biopsies. In the second group with 12-cores, a similar relationship could not be demonstrated. In cases with pure PIN pattern, especially the cribriform pattern may harden differential diagnosis with cribriform carcinoma. Again, HGPIN-like ductal adenocarcinoma may pose difficulties in differential diagnosis. The differential diagnostic problems are more easily overcome in recent years with the clarification of the diagnostic criteria of these entities and the help of immunohistochemical tests.

On the other hand, persistent HGPIN in repeat biopsies has been previously reported in 5-43\% of cases. According to Gokden et al.[8] experience, patients with persistent HGPIN should be biopsied sequentially; because their cancer detection risk in these cases is $41 \%$. In our study, the ratio of our cases who were diagnosed with isolated HGPIN (persistent HGPIN) in repeat biopsies was $19.3 \%$. It is noteworthy that this rate increased to $35.5 \%$ in cases with multifocal and bilateral HGPIN in their first biopsies. Existing data strongly suggest that this subgroup should be followed more closely with sequential bopsies in addition to clinical parameters. 
The decision to repeat prostate biopsy was at the discretion of the individual clinician, which is reflected in the large number of patients that did not undergo repeat biopsy during the study period. The concern of increased detection must be weighed against the risk of missing clinically significant malignancy.

The retrospective structure and the use of biopsy protocols, which are now known to provide insufficient sampling of the prostate gland in most of the studies, are among the limitations of the studies in the literature. However, the number of cores taken in the first biopsy is determined by the clinician who is monitoring the patient and may be affected by various clinical parameters such as PSA level, prostate volume, or DRE findings. Saturation biopsy protocols have been well studied in the repeat biopsy population, particularly in patients with elevated PSA levels. Increasing the number of samples and varying the distribution of biopsy sites has demonstrated a higher cancer detection rates, varying from $17 \%$ to $41 \%$ without an increase in clinically insignificant cancers. For that reasons, saturation biopsy protocol might be considered for the second and/or more repeat biopsies in multifocal and bilateral HGPIN and/or persistent HGPIN cases.

In our systematic literature review, the incidence of carcinoma on follow-up repeat biopsies ranged from $13 \%$ to $38 \%$ (average $25.6 \%$ ) for the largest series after the initial diagnosis of isolated HGPIN (totally 5580 isolated HGPIN cases with re-biopsies) on prostate needle biopsy. In these series, variable core numbers and follow-up intervals have been reported after the diagnosis of HGPIN on needle biopsy. The length of the follow-up period and the number of repeated biopsy sessions might also have affected the rates of carcinoma detection at repeat biopsy.

Although the necessity and timing of prostate biopsy after isolated HGPIN diagnosis remains controversial; current data suggest that increased sampling in repeat biopsies will enable more detection of prostate carcinoma in patients with a diagnosis of HGPIN. Concerns about increased detection of Gleason pattern score 6 cases must be weighed against the risk of missing clinically significant malignancies. When the Gleason pattern scores and Gleason grade groups of tumors were examined in cases with carcinoma in repeat biopsies; it was observed that Gleason pattern score $\geq 7$ (Grade group $\geq 2$ ) tumors, which are considered more important clinically, constitute $1 / 3^{\text {rd }}$ (33.3\%) of our cases in 12-core biopsy group.

The conclusions drawn may be somewhat limited by our relatively small study population and the retro- spective nature of our study. However, our data show that bilateral and multifocal HGPIN carries a significantly higher risk of carcinoma (25.8\%) in repeat biopsies. In addition to this, a much higher ratio (35.5\%) of both multifocal and bilateral persistent HGPIN has been re-diagnosed. Therefore, we also believe that sequential repeat biopsies are necessary in this specific high-risk population.

\section{Conclusion}

The incidence of isolated HGPIN in 6-core prostate needle biopsies evaluated in the first period 1996-2005 was $15 \%$, while carcinoma was found in the follow-up of $15.6 \%$ of the patients who had at least one repeat biopsy among the patients who were diagnosed with isolated HGPIN. In 12-core prostate needle biopsies evaluated in the period between 2006 and 2018, the incidence of isolated HGPIN was $4.2 \%$ and carcinoma was detected in $24 \%$ of these patients who underwent at least one repeat biopsy.

It was concluded that in cases with isolated HGPIN in prostate needle biopsies, the number of cores with HGPIN, bilaterality and prominent nuclear pleomorphism within the gland involved with HGPIN are histopathological findings that help to predict patients who will be diagnosed with carcinoma at repeat biopsies; a closer follow-up of cases in which these histopathological features were observed should be recommended with repeat biopsies as well as clinical parameters.

Note: The study was presented orally at the $4^{\text {th }}$ International Hipocrates Congress on Health Sciences on September 25, 2020.

Part of this study was presented as a poster at the $14^{\text {th }}$ Urooncology Congress held in Antalya on November 6-10, 2019.

Some findings of the cases in the first group of this study, although presented in Dr. Gupse Turan's academic thesis; it has not been published before in the same form.

Acknowledgement: The authors thank Professor Dr. Özdal Dillioğlugil and his team for the clinical follow-up of the cases.

Peer-review: Externally peer-reviewed.

Conflict of Interest: All authors declared no conflict of interest.

Ethics Committee Approval: The study was approved by the Kocaeli University Faculty of Medicine Non-Invasive Research Ethics Committee (No: GOAEK 2020/15.09, Date: 11/09/2020). 
Financial Support: None declared.

Authorship contributions: Concept - G.T., S.K.Ö.; Design - G.T., S.K.Ö.; Supervision - G.T., S.K.Ö.; Funding - None; Materials - G.T., S.K.Ö.; Data collection and/or processing - G.T., S.K.Ö.; Data analysis and/or interpretation - G.T., S.K.Ö.; Literature search - G.T., S.K.Ö.; Writing - G.T., S.K.Ö.; Critical review - G.T., S.K.Ö.

\section{References}

1. Bray F, Ferlay J, Soerjomataram I, Siegel RL, Torre LA, Jemal A. Global cancer statistics 2018: GLOBOCAN estimates of incidence and mortality worldwide for 36 cancers in 185 countries. CA Cancer J Clin 2018;68(6):394-424.

2. Epstein JI, Algaba F, Allsbrook WC, Bastacky S, Boccon-Gibod L, De Marzo AM, et al. Acinar adenocarcinoma. In: Eble JN, Sauter G, Epstein JL, Sesterhenn IA, editors. World Health Organisation Classification of Tumours. Pathology and Genetics of Tumours of the Urinary System and Male Genital Organs. Lyon: IARC Pres; 2004. p. 162-92.

3. McNeal JE, Bostwick DG. Intraductal dysplasia: A premalignant lesion of the prostate. Hum Pathol 1986;17(1):64-71.

4. Bostwick DG, Montironi R. Prostatic intraepithelial neoplasia and the origins of prostatic carcinoma. Pathol Res Pract 1995;191(9):828-32.

5. Tosoian JJ, Alam R, Ball MW, Carter HB, Epstein JI. Managing high-grade prostatic intraepithelial neoplasia (HGPIN) and atypical glands on prostate biopsy. Nat Rev Urol 2018;15(1):55-66.

6. Merrimen JL, Jones G, Walker D, Leung CS, Kapusta LR, Srigley JR. Multifocal high grade prostatic intraepithelial neoplasia is a significant risk factor for prostatic adenocarcinoma. J Urol 2009;182(2):485-90.

7. Bishara T, Ramnani DM, Epstein JI. High-grade prostatic intraepithelial neoplasia on needle biopsy: risk of cancer on repeat biopsy related to number of involved cores and morphologic pattern. Am J Surg Pathol 2004;28(5):629-33.

8. Gokden N, Roehl KA, Catalona WJ, Humphrey PA. High-grade prostatic intraepithelial neoplasia in needle biopsy as risk factor for detection of adenocarcinoma: Current level of risk in screening population. Urology 2005;65(3):538-42.

9. Girasole CR, Cookson MS, Putzi MJ, Chang SS, Smith JA Jr., Wells N, et al. Significance of atypical and suspicious small acinar proliferations, and high grade prostatic intraepithelial neoplasia on prostate biopsy: İmplications for cancer detection and biopsy strategy. J Urol 2006;175(3 Pt 1):929-33.

10. Kim TS, Ko KJ, Shin SJ, Ryoo HS, Song W, Sung HH, et al. Multiple cores of high grade prostatic intraepithelial neoplasia and any core of atypia on first biopsy are significant predictor for cancer detection at a repeat biopsy. Korean J Urol 2015;56(12):796-802.

11. Ongun Ş, Bozkurt O, Çömez K, Demir Ö, Aslan $\mathrm{G}$, Tuna B, et al. Rebiopsy results of the patients in which the first biopsy revealed high grade prostatic intraepithelial neoplasia. Bull Urooncol 2016;15:57-60.

12. Kronz JD, Allan CH, Shaikh AA, Epstein JI. Predicting cancer following a diagnosis of high-grade prostatic intraepithelial neoplasia on needle biopsy: Data on men with more than one follow-up biopsy. Am J Surg Pathol 2001;25(8):1079-85.

13. Dovey Z, Corbishley CM, Kirby RS. Prostatic intraepithelial neoplasia: A risk factor for prostate cancer. Can J Urol. 2005;12(Suppl 1):49-100.

14. Eggener SE, Roehl KA, Catalona WJ. Predictors of subsequent prostate cancer in men with a prostate specific antigen of 2.6 to $4.0 \mathrm{ng} / \mathrm{ml}$ and an initially negative biopsy. J Urol 2005;174(2):500-4.

15. Joniau S, Goeman L, Pennings J, Van Poppel H. Prostatic intraepithelial neoplasia (PIN): İmportance and clinical management. Eur Urol 2005;48(3):379-85.

16. Moore CK, Karikehalli S, Nazeer T, Fisher HA, Kaufman RP Jr., Mian BM. Prognostic significance of high grade prostatic intraepithelial neoplasia and atypical small acinar proliferation in the contemporary era. J Urol 2005;173(1):70-2.

17. Epstein JI, Herawi M. Prostate needle biopsies containing prostatic intraepithelial neoplasia or atypical foci suspicious for carcinoma: İmplications for patient care. J Urol 2006;175(3 Pt 1):820-34.

18. Herawi M, Kahane H, Cavallo C, Epstein JI. Risk of prostate cancer on first re-biopsy within 1 year following a diagnosis of high grade prostatic intraepithelial neoplasia is related to the number of cores sampled. J Urol. 2006;175(1):121-4.

19. El-Fakharany MM, Wojno KJ. Significance of high-grade prostatic intraepithelial neoplasia in the era of extended prostatic needle biopsies. Mod Pathol 2005;18:138A.

20.Sakr WGD, Srigley J, Troncoso P. Recent trends regarding the significance of high grade prostatic intraepithelial neoplasia HGPIN in predicting prostate cancer PCA on follow up biopsies. Is there a a change in confirming cancer diagnosis? A multi institutional study. Mod Pathol 2003;16:168A.

21. Lee MC, Moussa AS, Zaytoun O, Yu C, Jones JS. Using a saturation biopsy scheme increases cancer detection during repeat biopsy in men with high-grade prostatic intra-epithelial neoplasia. Urology 2011;78(5):1115-9.

22. Roscigno M, Scattoni V, Freschi M, Abdollah F, Maccagnano C, Galosi A, et al. Diagnosis of isolated high-grade prostatic intra-epithelial neopla- 
sia: Proposal of a nomogram for the prediction of cancer detection at saturation re-biopsy. BJU Int 2012;109(9):1329-34.

23. Pierson DM, Blute MJ, Zincke H. Prostate cancer biopsy and prostatectomy findings with cancer progression rates in men with initial needle biopsies positive for high-grade prostatic intraepithelial neoplasia. Mod Pathol 2004;17:172A.

24. Eskicorapci SY, Guliyev F, Islamoglu E, Ergen A, Ozen $\mathrm{H}$. The effect of prior biopsy scheme on prostate cancer detection for repeat biopsy population: Results of the 14-core prostate biopsy technique. Int Urol Nephrol 2007;39(1):189-95.

25. Schlesinger C, Bostwick DG, Iczkowski KA. Highgrade prostatic intraepithelial neoplasia and atypical small acinar proliferation: Predictive value for cancer in current practice. Am J Surg Pathol. 2005;29(9):1201-7.

26. Siever J, Orychak A, Trpkov K. Prostatic intraepithelial neoplasia, high grade (PIN): Pathologic and clinical findings on initial and repeat ten-core biopsy. Mod Pathol 2003;16:171A.

27. O’dowd GJ, Miller MC, Orozco R, Veltri RW. Analysis of repeated biopsy results within 1 year after a noncancer diagnosis. Urology. 2000;55(4):553-9.

28. Postma R, Roobol M, Schroder FH, van der Kwast TH. Lesions predictive for prostate cancer in a screened population: first and second screening round findings. Prostate 2004;61(3):260-6.
29. Davidson D, Bostwick DG, Qian J, Wollan PC, Oesterling JE, Rudders RA, et al. Prostatic intraepithelial neoplasia is a risk factor for adenocarcinoma: Predictive accuracy in needle biopsies. J Urol 1995;154(4):1295-9.

30. Wills ML, Hamper UM, Partin AW, Epstein JI. Incidence of high-grade prostatic intraepithelial neoplasia in sextant needle biopsy specimens. Urology 1997;49(3):367-73.

31. Lefkowitz GK, Sidhu GS, Torre P, Lepor H, Taneja SS. Is repeat prostate biopsy for high-grade prostatic intraepithelial neoplasia necessary after routine 12-core sampling? Urology 2001;58(6):999-1003.

32. Abdel-Khalek M, El-Baz M, Ibrahiem EH. Predictors of prostate cancer on extended biopsy in patients with high-grade prostatic intraepithelial neoplasia: A multivariate analysis model. BJU Int 2004;94(4):528-33.

33. Gallo F, Chiono L, Gastaldi E, Venturino E, Giberti C. Prognostic significance of high-grade prostatic intraepithelial neoplasia (HGPIN): Risk of prostatic cancer on repeat biopsies. Urology 2008;72(3):628-32.

34. Netto GJ, Epstein JI. Widespread high-grade prostatic intraepithelial neoplasia on prostatic needle biopsy: A significant likelihood of subsequently diagnosed adenocarcinoma. Am J Surg Pathol 2006;30(9):1184-8.

35. Lee MC, Moussa AS, Yu C, Kattan MW, Magi-Galluzzi C, Jones JS. Multifocal high grade prostatic intraepithelial neoplasia is a risk factor for subsequent prostate cancer. J Urol 2010;184(5):1958-62. 